\title{
Acute gonococcal arthritis: an unusual host and pathogen combination
}

\author{
D A Lewis, L M Pollock, J Randell, P Wilson, P G Kopelman
}

\begin{abstract}
A 76 year old man was admitted for evaluation and treatment of acute oligoarticular arthritis having recently returned from India. He was diabetic on admission. Neisseria gonorrhoeae was isolated from pus collected by arthrocentesis of his right knee and was subsequently found to be chromosomally resistant to penicillin. The isolate required proline for growth and expressed the protein 1B-1 serovar. These characteristics of host and pathogen are atypical in that gonococcal arthritis usually occurs in young women and is usually caused by $\boldsymbol{N}$ gonorrhoeae isolates exhibiting hypersusceptibility to penicillin, the arginine, hypoxanthine and uracil requiring auxotype, and the protein $1 \mathrm{~A}$ serotype. The patient responded well to therapy with oral ciprofloxacin and initial high dose penicillin.

(F Clin Pathol 1995;48:86-88)
\end{abstract}

Keywords: Neisseria gonorrhoeae, arthritis.

Case report

A 76 year old Indian man was admitted to hospital with a four day history of fever. Two days before admission, he developed acute joint swelling affecting both wrists and his right knee. His general practitioner prescribed co-trimoxazole and a non-steroidal antiinflammatory drug. He had returned from a five month stay in his native village in India one day before the start of his illness. During his trip, he practised and taught the art of yoga as a service to several members of his village. Initially, he denied any sexual contacts abroad and had not had sexual intercourse with his wife for several years. He had had no symptoms of dysuria, urethral discharge, or sore throat. His past medical history revealed bilateral osteoarthritis of both knees. There was nothing else of note in his medical history.

Physical examination revealed acute oligoarthritis and intermittent pyrexia with nightly peaks of $38^{\circ} \mathrm{C}$. Both wrist joints (figure) and the right knee joint were hot, tender, red, and swollen. He had vitiligo over both knees and a large effusion in the right knee joint. No skin lesions or urethral discharge were evident. The rest of the physical examination was normal.

His peripheral blood white cell count was raised at $14.7 \times 10^{9} / 1$. Biochemistry revealed diabetes and mildly impaired renal function: blood glucose $20.3 \mathrm{mmol} / 1$, urea $13.2 \mathrm{mmol} / \mathrm{l}$, and creatinine $209 \mu \mathrm{mol} / \mathrm{l}$. There were no abnormalities in the complement pathways.

Arthrocentesis of his right knee performed on admission yielded copious thick pus. Microscopy demonstrated numerous polymorphonuclear cells but no crystals. The Gram stain was initially reported as negative but was reviewed two days later following growth of Neisseria gonorrhoeae on culture and found to show a few intracellular Gram negative diplococci within the film. The isolate was identified by Gram stain, oxidase testing and utilisation of glucose but not maltose, lactose, or sucrose. Minimum inhibitory concentrations (MICs) for penicillin, ciprofloxacin, cefotaxime, and spectinomycin (table) were performed using an agar dilution method. The isolate did not produce $\beta$-lactamase on testing with nitrocefin. A growth requirement for proline was demonstrated by auxotyping. Serovar typing was performed using a standard panel of monoclonal antibodies directed against the gonococcal outer membrane protein 1 (Syva Palo Alto, USA); the isolate expressed the $1 \mathrm{~B}-1$ serovar.

Initial antibiotic treatment was instituted with intravenous benzylpenicillin $1.2 \mathrm{~g}$ and flucloxacillin $500 \mathrm{mg}$, both four times daily. With isolation of $N$ gonorrhoeae, flucloxacillin administration was stopped, the intravenous benzylpenicillin dose was increased to $2.4 \mathrm{~g}$ four times daily and treatment with oral ciprofloxacin $750 \mathrm{mg}$ twice daily was started pending susceptibility testing data. The patient's arthritis improved rapidly on this new regimen and he was discharged 12 days later with a further two week course of ciprofloxacin. He received regular analgesia with a non-steroidal anti-inflammatory agent together with regular physiotherapy whilst an inpatient. His diabetes was brought rapidly under control with a sliding scale insulin regimen and then substitution with oral gliclazide.

Five days after admission, he admitted to unprotected sexual intercourse over four

Minimum inhibitory concentrations for antibiotics tested against the gonococcal isolate

\begin{tabular}{ll}
\hline Antibiotic & $M I C(\mathrm{mg} / \mathrm{l})$ \\
\hline Penicillin & 0.5 \\
Ciprofloxacin & 0.004 \\
Cefotaxime & 0.06 \\
Spectinomycin & 32 \\
\hline
\end{tabular}


Gonococcal arthritis affecting the wrists and metacarpo-phalangeal joints of both hands.

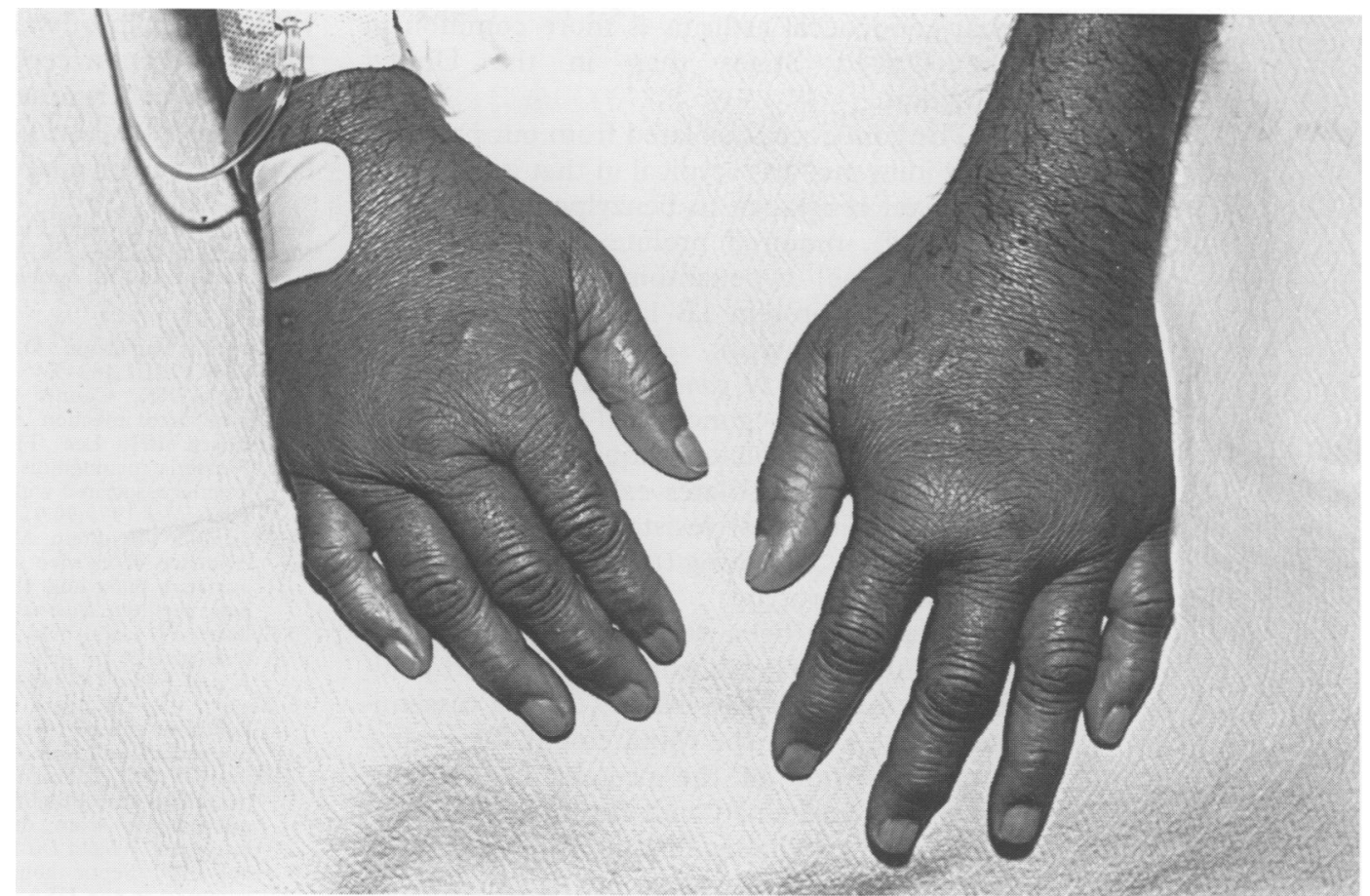

months with a 30 year old woman in India He had wanted to discover if he was still capable of fathering a child and had hoped for a son to carry on his life's work of yoga in India. He consented to screening for sexually transmitted diseases but urethral swabs failed to grow $N$ gonorrhoeae or Chlamydia trachomatis using culture techniques. This was not surprising given the fact that the patient had already received five days of benzylpenicillin therapy and three days of ciprofloxacin therapy. Serological screening for hepatitis B, hepatitis $\mathrm{C}$ and treponemal disease was negative at this time and at 10 weeks follow up The patient showed no evidence of antibodies to HIV at 10 weeks follow up. Appropriate contact tracing was performed.

\section{Discussion}

Gonococcal arthritis is often polyarticular and tends to affect the knees, wrists, small joints of the hands, ankles, and elbows. Most patients with disseminated gonococcal infection (DGI) are 15-35 years of age, single with more than one sexual partner, and likely to have had a sexually transmitted disease in the past. Race does not appear to be a significant factor except to the extent that it may reflect socioeconomic level, availability of health care, and degree of sexual promiscuity. In the United States, DGI occurs in $1-3 \%$ of patients harbouring $N$ gonorrhoeae. ${ }^{1}$ DGI occurs less frequently in the United Kingdom than in the United States and this clinical conundrum remains unexplained. In the preantibiotic era most patients with DGI were men, although many studies over the past 20 years have shown a female predominance of 78-97\%. ${ }^{12}$ Reasons underlying this shift include previous misdiagnosis of Reiter's syn- drome as DGI and prompt treatment of for infection include menses, second and third trimester pregnancy, and late acting (C5, C6, C7, C8) complement deficiency. ${ }^{3}$

Our 78 year old male patient was thus an atypical host and this case report emphasises the need to consider a gonococcal aetiology in all patients with acute septic arthritis. His age and uncontrolled diabetes may have contributed to an underlying immune deficiency allowing $N$ gonorrhoeae dissemination from the original site of inoculation. Chemotaxis and phagocytosis have long been known to be defective in hyperglycaemic diabetics and more recently it has been shown that neutrophil bactericidal activity may also be impaired. ${ }^{4}$

Early studies of DGI epidemiology in the United States showed that $N$ gonorrhoeae isolates from DGI patients were resistant to complement mediated bactericidal activity of normal human sera and unusually sensitive to in vitro killing by benzylpenicillin. ${ }^{5}$ Subsequent studies ${ }^{6}$ showed that these two characteristics of DGI isolates were associated with nutritional requirements (auxotrophy) for arginine, hypoxanthine, and uracil (AHU ${ }^{-}$strains). However, this requirement for AHU was later shown not to be a universal feature of all DGI isolates. ${ }^{7}$ Serotyping studies using monoclonal antibodies directed against the major gonococcal outer membrane protein (OMP 1) have revealed the dominant association of the protein 1A serotype with DGI prevalence. ${ }^{7}$ Most $\mathrm{AHU}^{-}$ gonococci belong to the protein 1A serotype as do most non-AHU ${ }^{-}$DGI isolates. It has been hypothesised that the different geographical prevalence of protein 1A serotypes may account for the observation uncomplicated gonorrhoea now. Risk factors 
that gonococcal arthritis is more common in the United States than in the United Kingdom. ${ }^{8}$

The gonococcus isolated from our patient's knee joint pus was atypical in that it exhibited low level resistance to benzylpenicillin (MIC $0.5 \mathrm{mg} / \mathrm{l}$ ), required proline for growth (but not arginine, hypoxanthine, or uracil) and expressed a protein 1B-1 serovar. In a study looking at auxotypes, serovars, and penicillin susceptibility of $N$ gonorrhoeae from 86 cases of disseminated gonococcal infection ${ }^{7}$ only $7 \%$ of DGI isolates required proline for growth, $19 \%$ of isolates exhibited intermediate susceptibility or resistance to benzylpenicillin (MIC $\geqslant 0.06 \mathrm{mg} / \mathrm{l}$ ), and only $7 \%$ had a protein 1B serotype.

There have been very few clinical reports of isolation of chromosomally mediated penicillin resistant $N$ gonorrhoeae from a disseminated site. ${ }^{9}$ Both the requirement for proline and expression of the protein 1B serotype have been associated with chromosomal resistance to benzylpenicillin in $N$ gonorrhoeae. ${ }^{10}$ Chromosomal resistance to penicillin in gonococci is due to mutations involving the pen $A$, mtr, pen $B$, and tet gene loci. It is increasing on a worldwide basis and is particularly prevalent in tropical regions such as India, South East Asia, and Africa. Therefore, we recommend that gonococcal infection acquired abroad be treated initially with either ciprofloxacin, spectinomycin, or a third generation cephalosporin such as cefotaxime or ceftriaxone. Antibiotic therapy may be changed to benzylpenicillin once susceptibility data are available.

We would like to thank Dr CA Ison at the Department of Medical Microbiology, St Mary's Hospital Medical School, London, for help with serotyping the isolate.

1 Barr J, Danielsson D. Septic gonococcal dermatitis $B M \mathcal{F}$ 1971;1:482-5.

2 Holmes KK, Counts GW, Beaty HN. Disseminated gonococcal infection. Ann Intern Med 1971;74:979-93.

3 Petersen BH, Lee TJ, Snyderman R, Brooks GF. Neisseria meningitidis and Neisseria gonorrhoeae bacNeisseria meningitidis and Neisseria gonorrhoeae bacteremia associated with C6,

4 Marhoffer W, Stein M, Schleinkofer L, Federlin K. Evidence of ex vivo and in vitro impaired neutrophil oxidative burst and phagocytic capacity in type 1 diabetes mellitus. Diabetes Res Clin Pract 1993;19:183-8.

5 Weisner PJ, Handsfield HH, Holmes KK. Low antibiotic resistance of gonococci causing disseminated infection. N Engl F Med 1973;288:1221-2.

6 Knapp JS, Holmes KK. Disseminated gonococcal infections caused by Neisseria gonorrhoeae with unique nutritional requirements. F Infect Dis 1975;132:204-8.

7 Bohnhoff M, Morello JA, Lerner SA. Auxotypes, penicillin susceptibility and serogroups of Neisseria penicillin susceptibility and serogroups of Neisseria gonorrhoeae from disseminated and
infections. $f$ Infect Dis 1986;154:225-30.

$8 \mathrm{Hook}$ EW. Septic gonococcal arthritis is much more common in the USA than in the UK. Br $\mathcal{F}$ Rheumatol 1990;29:283.

9 Strader KW, Wise CM, Wasilauskas BL, Salzer WL. Disseminated gonococcal infection caused by chromosomally mediated penicillin resistant organisms. Ann Intern Med 1986;104:365-6.

10 Rice RJ, Biddle JW, JeanLouis YA, Dewitt WE, Blount $\mathrm{JH}$, Morse SA. Chromosomally mediated resistance in Neisseria gonorrhoeae in the United States: results of surveillance and reporting, 1983-1984. F Infect Dis 1986;153:340-5. 